following combined kidney and nonmyeloablative marrow transplantation: in vivo and in vitro analyses. Am J Transplant. 2006;6(9):2121-2133.

13. Kawai T, et al. HLA-mismatched renal transplantation without maintenance immunosuppression. NEngl J Med. 2008;358(4):353-361.

14. Scandling JD, et al. Tolerance and chimerism after renal and hematopoietic-cell transplantation.
N Engl J Med. 2008;358(4):362-368.

15. Martinez-Llordella $M$, et al. Using transcriptional profiling to develop a diagnostic test of operational tolerance in liver transplant recipients. J Clin Invest. 2008;118(8):2845-2857.

16. Kruger B, Schroppel B, Murphy BT. Genetic polymorphisms and the fate of the transplanted organ. Transplant Rev (Orlando). 2008;22(2):131-140
17. Weaver TA, et al. Alefacept promotes co-stimulation blockade based allograft survival in nonhuman primates. Nat Med. 2009;15(7):746-749.

18. Heeger PS, et al. Pretransplant frequency of donorspecific, IFN-gamma-producing lymphocytes is a manifestation of immunologic memory and correlates with the risk of posttransplant rejection episodes. J Immunol. 1999;163(4):2267-2275.

\title{
Revisiting an old acquaintance: role for elF5A in diabetes
}

\author{
Joachim Hauber
}

Heinrich-Pette-Institute for Experimental Virology and Immunology, Hamburg, Germany.

\begin{abstract}
Dysfunction of pancreatic islet $\beta$ cells underlies both type 1 and type 2 diabetes and appears to result in part from the local release of proinflammatory cytokines. An improved understanding of the mechanisms that mediate islet responsiveness to proinflammatory cytokines may therefore expand our knowledge of the role of cytokine signaling in the development of diabetes, providing potential new targets for the development of therapeutics to protect pancreatic islets from inflammation. In this issue of the JCI, Maier and colleagues identify eukaryotic translation initiation factor 5A (eIF5A) as a critical regulator of the inflammatory response in mouse pancreatic islets. I believe these data provide new and important insights into the regulatory pathways that contribute to the development of diabetes and deepen our understanding of the function of the, so far, rather enigmatic cellular protein eIF5A.
\end{abstract}

Diabetes is a highly prevalent condition characterized by high blood glucose levels. Dysfunction and/or destruction of insulin-producing pancreatic islet $\beta$ cells underlies all forms of diabetes: in type 1 diabetes, $\beta$ cells are destroyed by an autoimmune response, while in type 2 diabetes, $\beta$ cell dysfunction and/or destruction is thought to arise because $\beta$ cells are unable to meet the increased demands for insulin. Despite these 2 distinct causes of $\beta$ cell dysfunction and/or destruction, emerging data suggest that in both cases local release of proinflammatory cytokines, such as IL- $1 \beta$, TNF- $\alpha$, and IFN- $\gamma$, is a central event. One pathway that contributes to the early pathogenesis of $\beta$ cell dysfunction in response to proinflammatory cytokines is NF- $\mathrm{\kappa B}$-mediated induction of the Nos2 gene, which encodes iNOS $(1,2)$. However, proinflammatory cytokine signaling ultimately leads to $\beta$ cell dysfunc-

Conflict of interest: The author has declared that no conflict of interest exists.

Citation for this article: J Clin Invest. 2010; 120(6):1806-1808. doi:10.1172/JCI43237. tion and death via both iNOS-dependent and -independent effects. Understanding the molecular mechanisms underlying the responsiveness of $\beta$ cells to proinflammatory cytokines will not only provide more insight into the pathogenesis of diabetes but also provide potential new targets for therapeutics to preserve pancreatic function. In this context, in this issue of the JCI, Maier et al. (3) identify eukaryotic translation initiation factor $5 \mathrm{~A}$ (eIF5A, formerly known as either IF-M2B $\alpha$ or eIF-4D) as a critical regulator of the response of mouse $\beta$ cells to proinflammatory cytokines.

\section{An initial career as translation initiation factor}

Functional characterization of eIF5A, purified by extraction of ribosomes from rabbit reticulocytes with buffers containing a high salt concentration, indicated that it had a stimulatory effect on the synthesis of methionyl-puromycin, an in vitro model reaction that mimics the formation of the first peptide bond during protein synthesis (4). Although it had, at that time, already been demonstrated that eIF5A had no effect on the translation of native rabbit globin mRNA (5), which actually argued against a role for eIF5A as an initiation factor in vivo, its activity in the methionylpuromycin assay led to this protein being considered a genuine translation initiation factor. For many years this notion seemed to be carved in stone, since the methionylpuromycin assay was the only available in vitro test system for analyzing eIF5A activity. However, the assumption that eIF5A directs translation initiation was challenged in the 1990s, when studies in the yeast Saccharomyces cerevisiae demonstrated that protein synthesis is only mildly affected by complete depletion of cellular eIF5A (6). This finding triggered the careful reevaluation of the reaction parameters of the methionyl-puromycin in vitro assay, and it became obvious that large amounts of eIF5A were required to obtain stimulation of methionyl-puromycin synthesis (7). In fact, routinely, 50-200 picomoles of eIF5A were used to obtain only 1-2 picomoles of reaction product, suggesting that this effect of eIF5A is an artifact of the assay system (7). More recent studies, employing polysome profiling in yeast and Drosophila cells, provided evidence that eIF5A participates in the elongation step of translation, rather than regulating the initiation of protein synthesis $(8,9)$. Whether this eIF5A activity affects the translation of all cellular mRNAs or occurs only in certain cell types and/or at specific physiological conditions remains to be elucidated.

\section{Posttranslational hypusine formation in elF5A}

eIF5A is unique because it is the only known cellular protein to contain the 


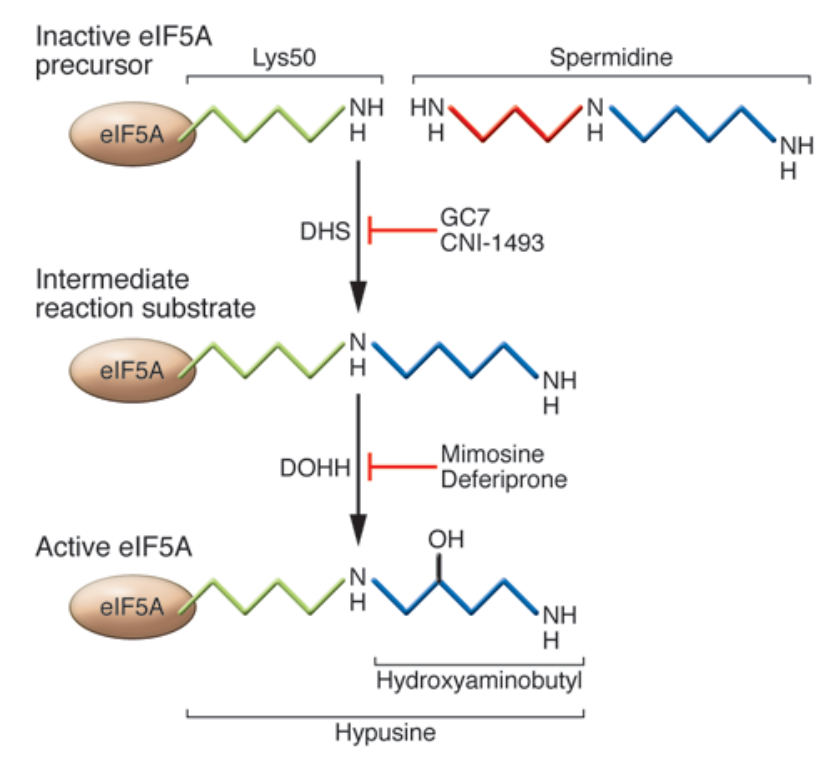

Figure 1

Hypusine modification of eIF5A. The enzymes DHS and DOHH catalyze hypusine modification of elF5A. DHS catalyzes the transfer of the aminobutyl moiety of the polyamine spermidine to the $\varepsilon-\mathrm{NH}_{2}$ group of Lys50 in the inactive elF5A precursor protein. This intermediate is subsequently hydroxylated by $\mathrm{DOHH}$, resulting in hypusine modification and the formation of active elF5A. DHS has been efficiently targeted by low-molecular-weight drugs, such as the spermidine analogue GC7 (24) and the multivalent guanylhydrazone CNI-1493 (14). The metalloenzyme DOHH has been targeted by iron-chelating compounds, such as mimosine and deferiprone (25).

unusual amino acid hypusine $\left(\mathrm{N}^{\varepsilon}-[4-\right.$ amino-2-hydroxybutyl]-lysine), a posttranslational modification that is formed by two subsequent enzymatic reactions (10) (Figure 1). In the first reaction, the aminobutyl moiety of the polyamine spermidine is transferred by deoxyhypusine synthase (DHS) to the $\varepsilon-\mathrm{NH}_{2}$ group of a single specific lysine (Lys50) in the inactive eIF5A precursor protein. This intermediate is subsequently hydroxylated by deoxyhypusine hydroxylase (DOHH), resulting in the active form of eIF5A. Inhibition of either DHS or DOHH prevents hypusine formation and thereby compromises eIF5A activity, because hypusine is required for many eIF5A functions. Since increased hypusine synthesis has been shown to occur in various activated and fast-dividing mammalian cells (reviewed in ref. 11), inhibition of hypusine formation (for example, by applying small-molecular-weight inhibitors of DHS and/or DOHH) has been suggested to provide a novel strategy to treat proliferative diseases (12).

\section{Participation of elF5A in inflammatory responses}

It was recently shown, in a murine model of severe sepsis, that the survival rate of LPS-challenged mice was substantially increased when eIF5A was inactivated by RNAi (13). Moreover, the serum concentration of proinflammatory cytokines was reduced in these animals (13), indicating that the hypusine-containing protein eIF5A participates in and can be essential to inflammatory responses. These data, and the fact that the Eif5 a gene localizes on mouse chromosome 11 within the type 1 insulin-dependent diabetes susceptibility (Idd4) locus, prompted Maier and coworkers to investigate whether eIF5A participates in the inflammatory cascade leading to pancreatic $\beta$ cell dysfunction and/or destruction (3). They analyzed the effects of inactivating eIF5A on islet function in the mouse low-dose streptozotocin (STZ) diabetes model as well as in isolated islets and various cell lines of rodent and human origin. They found that eIF5A was expressed in pancreatic islets and that animals in which eIF5A was depleted (by RNAi) or inhibited (by small-molecular-weight inhibitor) were resistant to $\beta$ cell loss and the development of hyperglycemia (3). Depletion of eIF5A in islets substantially reduced cytokineinduced synthesis of iNOS. Interestingly, the authors demonstrated that eIF5A indirectly promotes the translation of iNOS by binding, in an hypusine-dependent manner, to Nos 2 mRNA, mediating, in response to cytokine stimulation, its efficient nuclear export via a cellular pathway mediated by chromosome region maintenance 1 (CRM1, also known as exportin1). This suggests that targeting the hypusine modification of eIF5A by interfering with DHS activity may represent a novel therapeutic strategy to protect pancreatic islets from inflammation during the development of diabetes.

\section{Linking specific mRNAs to the translational machinery}

Clearly, the predominant activity of eIF5A is connected to its role in cytoplasmic regulation of translation, an activity that depends on the presence of its hypusine modification $(8,9)$. However, a number of independent studies have indicated that, particularly in mammalian cells, eIF5A also exhibits activity in the nucleus (14-16). For example, it has been shown that eIF5A participates in the nucleocytoplasmic transport of unspliced and incompletely spliced HIV-1 mRNAs (see ref. 14 and references therein), which are translocated across the nuclear envelope via the CRM1 pathway. CRM1 is a member of the importin $\beta$ superfamily of transport receptors and mediates a rather atypical mRNA transport route; its predominant function is to mediate the translocation of ribosomal subunits, rRNA, and U snRNA across the nuclear envelope (17). It has been shown also that eIF5A can colocalize and interact with another importin $\beta$ family member, the transport receptor exportin4 (16). In sum, these data indicate a nuclear activity for eIF5A that conceivably is connected to cellular mRNA metabolism. Supporting this notion, it has been reported that eIF5A itself has RNA-binding properties (18). Moreover, in the lower eukaryote S. cerevisiae, eIF5A appears to affect mRNA turnover/degradation $(19,20)$, a process that is closely linked to nucleocytoplasmic mRNA transport.

The present study by Maier and colleagues (3) identifies hypusine-modified eIF5A as a critical regulator of the nuclear export of Nos 2 mRNA, further substantiating the notion that eIF5A has a nuclear function. In their model of Nos2 translation, the authors suggest that Nos 2 transcripts are directly recognized in the nucleus by hypusine-modified eIF5A and are subsequently exported to the cytoplasm in a CRM1- and eIF5A-dependent manner. The nuclear recruitment of eIF5A into the Nos 2 mRNA ribonucleoprotein complex may additionally promote cytoplasmic 
translation of iNOS, which, in turn, leads to suppression of ATP generation and to the eventual inhibition of insulin release. As their experiments demonstrate, inhibition of hypusine synthesis indeed interferes with the inflammatory response in pancreatic islets (3). In sum, this study unveils hypusine-modified eIF5A to be a so far unrecognized player in the inflammatory cascades that are triggered by cytokine signaling during the development of diabetes. It thereby provides a potential new cellular target for the development of islet-preserving therapeutics.

\section{Future perspectives}

In more general terms, one may speculate that hypusine-containing eIF5A affects the translation of a subset of specific cellular mRNAs. In this context, eIF5A may specify a transport pathway that, upon activation of the cell, assures the timely and efficient synthesis of proteins with regulatory function (e.g., iNOS). It is assumed that CRM1 (and/or exportin4) provides a fast track to the cytoplasm for eIF5A-bound mRNAs, thereby ensuring a kinetic advantage in nuclear export over the vast majority of cellular transcripts, which are commonly handled by the unrelated general mRNA export receptor TAP/NXF1 (21). In support of this hypothesis, it has been previously shown that a small number of cellular transcripts is transported by CRM1, particularly in activated lymphocytes (22). These transcripts include, for example, the mRNA encoding CD83, a surface protein primarily expressed on mature immunecompetent dendritic cells (23). Strikingly, it has been demonstrated that inactivation of eIF5A (by DHS inhibition) results in nuclear trapping of CD83-encoding transcripts (15), a result that is reminiscent of the data shown for the Nos 2 mRNA in the study by Maier and colleagues (3). It is therefore conceivable that, as the complexity of organisms has increased during evolution, eIF5A has gained activities in addition to its role in promoting translation. As the present study strongly suggests (3), in mammalian cells, these additional eIF5A activities appear to regulate mRNA processing, presumably by efficiently directing specific mRNAs to the translational machinery. Such a mechanism may facilitate the rapid response of resting cells to extracellular stimuli and, apparently, can be targeted in a highly specific manner by blocking hypusine formation.

\section{Acknowledgments}

The author is supported within the framework initiative "Innovative Therapies" (grant 01GU0715) by the Federal Ministry of Education and Research (BMBF). The HeinrichPette-Institute is a member of the Leibniz Gemeinschaft (WGL) and is supported by the Free and Hanseatic City of Hamburg and the Federal Ministry of Health.

Address correspondence to: Joachim Hauber, Heinrich-Pette-Institute for Experimental Virology and Immunology, Martinistrasse 52, 20251 Hamburg, Germany. Phone: 49.40.48051.241; Fax: 49.40.48051.184; E-mail: joachim.hauber@ hpi.uni-hamburg.de.

1. Leist M, et al. Inhibition of mitochondrial ATP generation by nitric oxide switches apoptosis to necrosis. Exp Cell Res. 1999;249(2):396-403.

2. Koster JC, Marshall BA, Ensor N, Corbett JA, Nichols CG. Targeted overactivity of beta cell K(ATP) channels induces profound neonatal diabetes. Cell. 2000;100(6):645-654.

3. Maier B, et al. The unique hypusine modification of eIF5A promotes islet $\beta$ cell inflammation and dysfunction in mice. J Clin Invest. 2010; 120(6):2156-2170

4. Kemper WM, Berry KW, Merrick WC. Purification and properties of rabbit reticulocyte protein synthesis initiation factors M2Balpha and M2Bbeta. J Biol Chem. 1976;251(18):5551-5557.

5. Schreier MH, Erni B, Staehelin T. Initiation of mammalian protein synthesis. I. Purification and characterization of seven initiation factors. $J \mathrm{Mol}$ Biol. 1977;116(4):727-753.

6. Kang HA, Hershey JW. Effect of initiation factor eIF-5A depletion on protein synthesis and proliferation of Saccharomyces cerevisiae. J Biol Chem. 1994;269(6):3934-3940.

7. Kang HA, Schwelberger HG, Hershey JWB. Effect of initiation factor eIF-5A depletion on cell proliferation and protein synthesis. In: Brown AJP, Tuite MF, McCarthy JEG, eds. Protein synthesis and targeting in yeast. Berlin, Germany: Springer Verlag; 1993:123-129.

8. Saini P, Eyler DE, Green R, Dever TE. Hypusinecontaining protein eIF5A promotes translation elongation. Nature. 2009;459(7243):118-121.
9. Patel PH, Costa-Mattioli M, Schulze KL, Bellen HJ. The Drosophila deoxyhypusine hydroxylase homologue nero and its target eIF5A are required for cell growth and the regulation of autophagy. J Cell Biol. 2009;185(7):1181-1194.

10. Park MH. The post-translational synthesis of a polyamine-derived amino acid, hypusine, in the eukaryotic translation initiation factor $5 \mathrm{~A}$ (eIF5A). J Biochem. 2006;139(2):161-169.

11. Park MH, Wolff EC, Folk JE. Is hypusine essential for eukaryotic cell proliferation? Trends Biochem Sci. 1993;18(12):475-479.

12. Caraglia $\mathrm{M}$, et al. The role of eukaryotic initiation factor $5 \mathrm{~A}$ in the control of cell proliferation and apoptosis. Amino Acids. 2001;20(2):91-104.

13. Moore CC, et al. Eukaryotic translation initiation factor 5A small interference RNA-liposome complexes reduce inflammation and increase survival in murine models of severe sepsis and acute lung injury. J Infect Dis. 2008;198(9):1407-1414.

14 . Hauber I, et al. Identification of cellular deoxyhypusine synthase as a novel target for antiretroviral therapy. J Clin Invest. 2005;115(1):76-85.

15 . Kruse M, et al. Inhibition of CD83 cell surface expression during dendritic cell maturation by interference with nuclear export of CD83 mRNA. J Exp Med. 2000;191(9):1581-1590.

16. Lipowsky G, et al. Exportin 4: a mediator of a novel nuclear export pathway in higher eukaryotes. EMBO J. 2000;19(16):4362-4371.

17. Hutten S, Kehlenbach RH. CRM1-mediated nuclear export: to the pore and beyond. Trends Cell Biol. 2007;17(4):193-201.

18. Xu A, Chen KY. Hypusine is required for a sequence-specific interaction of eukaryotic initiation factor $5 \mathrm{~A}$ with postsystematic evolution of ligands by exponential enrichment RNA.J Biol Chem. 2001;276(4):2555-2561.

19. Zuk D, Jacobson A. A single amino acid substitution in yeast eIF-5A results in mRNA stabilization. EMBO J. 1998;17(10):2914-2925.

20. Schrader R, Young C, Kozian D, Hoffmann R, Lottspeich F. Temperature-sensitive eIF5A mutant accumulates transcripts targeted to the nonsense-mediated decay pathway. J Biol Chem. 2006; 281(46):35336-35346.

21. Carmody SR, Wente SR. mRNA nuclear export at a glance. J Cell Sci. 2009;122(Pt 12):1933-1937.

22. Schütz S, Chemnitz J, Spillner C, Frohme M, Hauber J, Kehlenbach RH. Stimulated expression of mRNAs in activated $T$ cells depends on a functional CRM1 nuclear export pathway. J Mol Biol. 2006; 358(4):997-1009.

23. Prechtel AT, Steinkasserer A. CD83: an update on functions and prospects of the maturation marker of dendritic cells. Arch Dermatol Res. 2007; 299(2):59-69.

24. Wolff EC, Kang KR, Kim YS, Park MH. Posttranslational synthesis of hypusine: evolutionary progression and specificity of the hypusine modification. Amino Acids. 2007;33(2):341-350.

25. Andrus L, et al. Antiretroviral effects of deoxyhypusyl hydroxylase inhibitors: a hypusine-dependent host cell mechanism for replication of human immunodeficiency virus type 1 (HIV-1). Biochem Pharmacol. 1998;55(11):1807-1818. 


\title{
Caught red-handed: uric acid is an agent of inflammation
}

\author{
Yan Shi \\ Immunology Research Group and Department of Microbiology \& Infectious Diseases, University of Calgary, Calgary, Alberta, Canada.
}

\begin{abstract}
Inflammation occurs in response to both pathogenic insult and tissue damage under sterile conditions, with the latter contributing to the pathogenesis of many diseases. Although several endogenous substances, including uric acid, have been suggested to alert the body to danger and to stimulate inflammation, little is known about their contribution to such responses in vivo. In this issue of the JCI, Kono et al. use newly generated mice with reduced levels of uric acid to investigate its role as an endogenous signal of tissue damage in inflammatory responses to hepatic injury. They find that uric acid is released from dying tissues and induces inflammation to cell death but is not involved in the response to microbial molecules or sterile irritant particles. I believe this to be the first report of an endogenous danger signal acting as a physiological regulator of inflammation.
\end{abstract}

PAMPs vs. DAMPs: evidence needed

Charles Janeway proposed the concept of innate immunity over 20 years ago, when he suggested that APCs expressed germline-encoded receptors that sensed microbial products (pathogen-associated molecular patterns [PAMPs]) and that triggering of these receptors initiated inflammatory and adaptive immune responses (1). Several years later, Polly Matzinger developed her danger hypothesis, whereby she proposed that APCs can sense endogenous signals released by damaged cells/tissues (signals she later termed damage-associated molecular patterns [DAMPs]) to trigger an immune response in the absence of PAMPs (i.e., under sterile conditions) (2). Generally, it is recognized that the two theories deal with two different sets of immune surveillance: external (exogenous) insults are detected by the innate immune system, while internal (endogenous) danger is the remit of Matzinger's hypothesis. The two concepts are symmetrical and complementary. However, their fates have turned out to be quite different. Janeway's hypothesis has been developed into the robust system of innate immune recognition, with many of the molecular intricacies of receptors, ligands, and signaling mechanisms described in the finest details (3). By contrast, the concept of endogenous adjuvants

Conflict of interest: The author has declared that no conflict of interest exists.

Citation for this article: J Clin Invest. 2010; 120(6):1809-1811. doi:10.1172/JCI43132. or danger signals is less well developed and, to a large extent, remains a conceptual discussion. Lack of elegance in the danger theory itself is certainly not the reason. Rather, the danger hypothesis thus far has not gathered the robust experimental support and verification that Janeway's proposal of innate immunity has relished.

Currently, there are several endogenous substances that are candidate DAMPs, including high-mobility group box 1 (HMGB1), heat shock proteins, and uric acid (4), and it is thought these are the substances released from injured cells that mediate inflammatory responses in the absence of PAMPs. While some of these have been found to stimulate sterile inflammatory responses in vitro, it has remained an open question as to whether any of them has a nonredundant role in a physiological/pathological situation. In this issue of the JCI, Kono et al. offer support for the idea that uric acid levels regulate sterile inflammation in vivo (5).

\section{An in vivo model of uric acid- induced sterile inflammation}

Uric acid is the final product of purine metabolism in humans and higher primates. It has been known for a long time to have a role in gout, since this painful inflammatory condition is the result of uric acid (monosodium urate [MSU]) crystals precipitating in joints and capillaries (6). Its association with other forms of inflammation and immune regulation was not apparent until it was found to serve as an adjuvant in CTL responses against particulate antigens (7). In the last several years, we have learned a number of features of this simple catabolite. First, it appears that uric acid has to be in the range of supersaturation $(>70 \mu \mathrm{g} / \mathrm{ml})$ to exert its adjuvant effect, implying that it elicits this effect as a crystal. Cell death results in local supersaturation of uric acid, as cytolysis generates large quantities of purines from RNA and DNA. Second, crystalline MSU binds to lipids on the surface of APCs and signals through the NACHT, LRR, and PYD domain-containing protein 3 (NLRP3) inflammasome to produce IL-1 $\beta(8,9)$. Third, preformed MSU crystals appear to promote tumor rejection (10). Fourth, depletion of uric acid reduces the effect of alum in vaccines (11). Despite these findings, which hinted at the ability of uric acid to act as a DAMP, to systematically study the effect of uric acid, an animal model is needed, as pharmacological inhibition of uric acid is short lived. Furthermore, uricase-deficient mice die from kidney failure before weaning, unless rescued by injection of the enzyme conjugated to polyethylene glycol (12). The work of Kono et al. (5) therefore introduces what I believe to be the first viable animal models generated for the purpose of immunological research on uric acid. Using the mice they generated, they confirmed that uric acid can participate in the induction of sterile inflammation in vivo.

Kono et al. (5) approached the issue of how to modulate uric acid levels from the opposite direction of uricase deficiency, reducing uric acid levels by introducing transgenes to increase uricase expression. Several details of their design are worth noting. In mammals, uricase is expressed only in the liver. In this study, one version of the transgene is fused to a viral signal sequence that promotes extracellular secretion, increasing its availability in interstitial fluids. The other construct targets uricase to peroxisomes, where it reduces intracellular pools of uric acid, and its effects are only apparent upon cytolysis. Both transgenes are under the control 